\title{
Sobrecarga de Cuidadores de Indivíduos com a Doença de Parkinson: Atividade Física, Qualidade de Vida e Sintomas Depressivos
}

\section{Caregiver's Burden of Individuals with Parkinson Disease: Physical Activity, Quality of Life and Depressive Symptoms}

\author{
Nataly Souza Severo' \\ Jessica Moratelli ${ }^{2}$ \\ Zenite Machado ${ }^{3}$
}

Adriana Coutinho de Azevedo Guimarães ${ }^{4}$

\section{RESUMO}

Objetivo: Investigar o nível de atividade física, a qualidade de vida e os sintomas depressivos, de acordo com a sobrecarga de cuidadores de indivíduos com a doença de Parkinson. Metodologia: Estudo de delineamento transversal com 48 cuidadores, ( $45 \pm 16$ anos), sendo 44 do sexo feminino. Utilizouse um questionário autoaplicável, dividido em cinco partes: informações gerais; IPAQ; WHOQOL; Burden Interview e sintomas depressivos. Resultados: $59 \%$ dos cuidadores eram formais, trabalhavam em regime de 12 horas $(p=0,020)$, sem vínculo familiar $(p=0,007)$ e possuíam sobrecarga leve ou mesmo ausente. Observou-se que não houve diferenças significativas entre os grupos, porém os cuidadores com sobrecarga leve ou ausente apresentaram escores mais elevados de qualidade de vida, bem como maiores percentuais de sintomas depressivos leves ou ausentes e grande parte dos cuidadores foi classificada como insuficientemente ativos $(95,8 \%)$. Conclusão: Cuidadores considerados insuficientemente ativos, sendo os formais com uma qualidade de vida melhor e sintomas depressivos leves ou ausentes, mesmo não existindo diferenças significativas, necessitando de mais pesquisas com este grupo.

\section{DESCRITORES}

Atividade Física. Cuidadores. Doença de Parkinson. Qualidade de Vida. Sintomas Depressivos.

\begin{abstract}
Objetivo: To investigate the relationship between the level of physical activity, quality of life and depressive symptoms of caregivers of individuals with Parkinson's disease. Methodology: Cross-sectional study with 48 caregivers, ( $45 \pm 16$ years) being 44 females. A self-administered questionnaire was used, divided into five parts: general information; IPAQ short version; WHOQOL; Burden Interview and depressive symptoms. Results: $59 \%$ of the participants were formal caregivers; they worked 12 hours $(p=0.020)$ without a family bond $(p=0.007)$ and had moderate or even minimal overload compared to informal caregivers. It was observed that there were no significant differences between the groups, but the caregivers with light or no overload presented higher quality of life scores, as well as higher percentages of moderate or no depressive symptoms and practically all caregivers were listed as insufficiently active (95.8). Conclusion: Considered insufficiently active, being the formal ones with a better quality of life with moderate or without depressive symptoms, even though there were no significant differences, maintaining the need for more research with this group.
\end{abstract}

\section{DESCRIPTORS}

Physical Activity. Caregivers. Parkinson's Disease. Quality of Life. Depressive symptoms.

${ }^{1}$ Graduada em Educação Física pelo Centro de Ciências da Saúde e do Esporte da Universidade do Estado de Santa Catarina. Florianópolis, SC, Brasil. ORCID: 0000-0002-8837-4298.

${ }^{2}$ Doutoranda em Ciências do Movimento Humano pelo Centro de Ciências da Saúde e do Esporte da Universidade do Estado de Santa Catarina. Florianópolis, SC, Brasil. ORCID: 0000-0003-2007-4552.

${ }^{3}$ Professora Doutora do Departamento de Educação Física e do Programa de Pós-Graduação em Ciências do Movimento Humano do Centro de Ciências da Saúde e do Esporte da Universidade do Estado de Santa Catarina. Florianópolis, SC, Brasil. ORCID: 0000-00024569-8460.

${ }^{4}$ Professora Doutora do Departamento de Educação Física e do Programa de Pós-Graduação em Ciências do Movimento Humano do Centro de Ciências da Saúde e do Esporte da Universidade do Estado de Santa Catarina. Florianópolis, SC, Brasil. ORCID: 0000-00015167-2921. 
A doença de Parkinson (DP) é uma doença neurodegenerativa progressiva que afeta principalmente pessoas a partir da quinta década de vida ${ }^{1,2}$. É uma alteração neurológica, que provoca a redução dos neurônios dopaminérgicos da substância negra $^{3}$ e sua prevalência tende a crescer de acordo com a idade ${ }^{4}$. Os sintomas são agravados de acordo com o avanço da idade ${ }^{2,4}$. Os sintomas mais comuns dessa doença são a bradicinesia, a rigidez, o tremor, a instabilidade postural, os distúrbios da marcha e a fadiga ${ }^{5-7}$. Assim, interfere nos movimentos voluntários e automáticos, levando ao isolamento social, perda de interesse por atividades de lazer, aumentando a dependência para as atividades de vida diária, a perda de autonomia e, consequentemente, a redução da qualidade de vida ${ }^{8,9}$.

Nesse sentido, o cuidador torna-se extremamente importante para o individuo com DP, pois são eles que assumem o compromisso com os cuidados básicos e dão suporte para as tarefas do dia a dia ${ }^{10}$. Contudo, o cuidador acaba encarando o desafio de ajustar a sua vida, de acordo com os cuidados que devem ser prestados com o individuo com DP, muitas vezes deixando de lado a sua profissão, as suas atividades de lazer, o cuidado consigo mesmo, prejudicando a sua qualidade de vida ${ }^{11}$. Por estes motivos, os cuidadores apresentamse com estresse, sintomas depressivos, ansiedade e sobrecarga ${ }^{12,13}$.

Assim sendo, a sobrecarga dos cuidadores é bastante relatada, devido ao alto nível de estresse e cuidados exigidos com os pacientes ${ }^{11,14}$. Dessa maneira, a prática da atividade física desempenha um importante papel na promoção da saúde, na percepção de qualidade de vida, nos sintomas depressivos e no aumento da expectativa de vida ${ }^{14}$. A atividade física traz inúmeros benefícios tanto nos aspectos físicos e psicológicos dos cuidadores, reestabelecendo o bem-estar físico, social, mental, proporcionando um retorno positivo nos cuidados ao indivíduo ${ }^{15}$.

Além do que, é vital ter uma atenção especial com os cuidadores, visto que eles necessitam de assistência, auxílio, instrução e cuidado, que podem trazer melhora da qualidade de vida e influenciar positivamente nos cuidados do indivíduo com $\mathrm{DP}^{16}$. Tendo em vista a importância desta temática, este estudo teve como objetivo investigar o nível de atividade física, a qualidade de vida e os sintomas depressivos, de acordo com a sobrecarga de cuidadores de indivíduos com a doença de Parkinson.

\section{METODOLOGIA}

O estudo analítico de delineamento transversal, aprovado no Comitê de Ética em Pesquisa em Seres Humanos (CEPSH) da UDESC, protocolo $n^{\circ} 2.070 .849$, em maio de 2017. Os dados foram coletados entre maio e outubro de 2017 e fizeram parte deste estudo 48 cuidadores, com média de idade de $45 \pm 16$ anos, sendo 44 do sexo feminino; todos maiores de 18 anos, residentes em Florianópolis e São José.

Para o critério de inclusão, foi considerado o domicílio em Florianópolis e São José, com idade superior a 18 anos e que trabalhasse como cuidador exclusivo de um indivíduo com a doença de Parkinson, podendo ser formal (profissionais e instituições 
que realizam atendimento sob a forma de prestação de serviços) ou informal (membro da família ou pessoa próxima do indivíduo). Como critério de exclusão determinouse o nível de escolaridade, classificada como analfabeta, devido à necessidade de compreensão do questionário.

Os dados foram coletados por meio de um questionário estruturado individualmente, aplicado por duas pesquisadoras do Laboratório de Pesquisa em Lazer e Atividade Física - LAPLAF, após treinamento prévio. A pesquisa foi inicialmente realizada no Centro Catarinense de Reabilitação (CCR) e, posteriormente, com visitas às residências dos cuidadores, com duração média de 30 minutos para a aplicação e resposta dos questionários. No total, foram entregues 58 questionários aos cuidadores, no qual 48 foram respondidos; 2 participantes se recusaram a participar da pesquisa e 8 questionários não retornaram até o final do estudo. Todos os participantes foram convidados a participar voluntariamente do estudo, após a assinatura do Termo de Consentimento Livre e Esclarecido.

\section{Instrumentos de pesquisa}

O questionário utilizado foi composto por instrumentos validados:

a) Informações gerais: caracterização dos participantes quanto à idade, a escolaridade, o tipo de cuidador, o regime de plantão, a remuneração, o seu vínculo com o paciente (foi agrupado em três vínculos: esposa (o); filha (o) e sem vínculo, uma vez que nos demais vínculos não houve preenchimento), atividades que realiza e a severidade da doença do paciente. A severidade da doença foi avaliada pela escala de Hoehn e Yahr ${ }^{17}$, que compreende cinco estágios de classificação para avaliar a severidade da DP e abrange medidas globais de sinais e sintomas que permitem classificar o indivíduo quanto ao nível de incapacidade. Foi agrupada, em nível leve e moderada, em função da não existência de pacientes com o nível severo da doença.

As medidas antropométricas: massa corporal e estatura (autorreferida) para o cálculo do Índice de massa corporal - IMC (peso/estatura ${ }^{2}$ ). Para classificação do IMC (estado nutricional) foi utilizado o protocolo da Organização Mundial de Saúde (2004), ou seja, magreza $(\mathrm{IMC}<18,5)$; peso normal (IMC 18,5-24,9); sobrepeso (IMC 25,0-29,9); pré-obesidade e obesidade $(\mathrm{IMC}>30,0)$. Para fins estatísticos, o IMC foi agrupado em peso normal (magreza/peso normal) e acima do peso (sobrepeso e obesidade), pelo número reduzido em algumas variáveis.

b) Atividade física: por meio do questionário internacional de atividade física (IPAQ - versão curta) ${ }^{18}$. O IPAQ é um instrumento autoaplicável, composto por seis itens, que procura verificar o número de vezes em que o sujeito praticou pelo menos 10 minutos contínuos de caminhada, atividade física moderada e vigorosa, na última semana, em diversos envolvimentos, nomeadamente, laboral, doméstico, lazer, recreativo e desportivo.

c) Qualidade de vida - avaliada por meio do questionário abreviado World Health Organization Quality of Life (WHOQOL), que se encontra adaptado para português do Brasili19. Trata-se de um questionário composto por 26 questões (dos 100 originais), que se referem às duas últimas semanas. 
Contém 24 questões que abrangem: o domínio físico (dor física, energia, locomoção, atividades da vida diária, tratamento médico, trabalho), psicológico (sentimentos positivos, concentração, autoestima, autoimagem, sentimentos negativos, espiritualidade), social (relações pessoais, suporte social, atividade sexual) e meio ambiente (segurança física, habitação, recursos financeiros, serviço de saúde, informações, lazer, ambiente físico e transporte,) e mais duas questões gerais sobre qualidade de vida. Os valores são computados em escores que variam de 0 (péssimo), a 100\% (excelente).

d) Sobrecarga - Avaliada por meio do questionário Burgen Interview, que se encontra adaptado para o português ${ }^{20}$. É um instrumento de 22 itens, pelo qual se avalia a sobrecarga do cuidador em diferentes níveis. Para cada item, os cuidadores devem indicar a frequência (nunca, raramente, às vezes, com bastante frequência ou quase sempre). A Burden Interview é pontuada adicionando-se as respostas numeradas dos itens individuais. As pontuações mais altas indicam um maior sofrimento do cuidador; escores de 0-20: ausência de sobrecarga; escores de 21-40: sobrecarga moderada; escores de 41-60: sobrecarga moderada a severa; escores de 61-88: sobrecarga severa. Para fins estatísticos, a sobrecarga foi agrupada em sobrecarga leve ou ausente e sobrecarga moderada a severa, pelo número reduzido de respostas nas variáveis, além disso, não ocorreram participantes na variável sobrecarga severa.

e) Sintomas Depressivos - O Inventário de Depressão de Beck (BDI), trata-se de um questionário de autorrelato desenvolvido originariamente por Beck et al. ${ }^{21}$. Foi validado no Brasil por Cunha et al. ${ }^{22}$ que manteve a escala original de 21 itens, com escore de zero a três, com quatro alternativas, que correspondem a níveis crescentes de gravidade de depressão. A soma dos escores dos itens individuais fornece um escore total, onde o maior escore é 63 , que indica alto grau de depressão e o escore mais baixo é o zero, que corresponde à ausência de depressão. A interpretação dos escores foi realizada de acordo com a padronização: escores de zero a 11, sem depressão ou depressão mínima; escores de 12 a 19, depressão leve; escores de 20 a 35, depressão moderada; escores de 36 a 63, depressão grave ${ }^{22}$. Para fins de análise estatística, os sintomas depressivos foram agrupados em nível leve/sem depressão e depressão moderada/grave, sendo que não existiu nenhum caso de sintoma depressivo grave.

Os dados foram analisados por meio do SPSS - IBM versão 20.0. Inicialmente, foi realizada uma análise descritiva (média, desvio padrão e percentagem) para conhecimento dos dados e, posteriormente, uma associação das variáveis independentes (tipo de cuidador; regime de plantão; remuneração; vínculo familiar; cuidador por opção; tempo com cuidador; principais atividades) com a sobrecarga, utilizando-se os testes do Quiquadrado e Exato de Fisher; para associar a qualidade de vida e os sintomas depressivos com a sobrecarga, aderiu-se ao uso do Exato de Fisher e o Teste t de Student, para amostras independentes. O nível de significância adotado nas análises foi de $5 \%$. 


\section{RESULTADOS}

Participaram do estudo 48 cuidadores, com nível de escolaridade médio (62,5\%). No momento da pesquisa, $63 \%$ apresentavam excesso de peso e $96 \%$ estavam insuficientemente ativos. Estes cuidadores prestavam atendimento a indivíduos com DP com nível leve (29\%), moderado $(67,8 \%)$ e severo $(3,2 \%)$.

A Tabela 1 mostra a caracterização dos cuidadores, discriminada de acordo com a sobrecarga dos mesmos. Pode-se observar que os cuidadores formais $(p=0,034)$, em regime de 12 horas $(p=0,020)$ e sem vínculo familiar $(p=0,007)$ possuíam sobrecarga leve ou mesmo ausência desta, em relação aos cuidadores informais (familiares). As demais variáveis não apresentaram diferenças significativas.

$\mathrm{Na}$ Tabela 2 encontram-se os resultados referentes aos domínios da qualidade de vida e dos sintomas depressivos, de acordo com os grupos de sobrecarga. Observou-se que não ocorreram diferenças significativas entre os grupos, porém, destaca-se que os cuidadores com sobrecarga leve ou ausente apresentaram escores mais elevados de qualidade de vida, bem como maiores percentuais de sintomas depressivos leves ou ausentes. Praticamente,

Tabela 1. Caracterização dos cuidadores participantes do estudo. Florianópolis. 2017.

\begin{tabular}{|c|c|c|c|c|}
\hline Variáveis & $\begin{array}{l}\text { Total } \\
\text { n (\%) }\end{array}$ & $\begin{array}{c}\text { Sobrecarga leve } \\
\text { ou ausência } \\
(\%)\end{array}$ & $\begin{array}{c}\text { Sobrecarga } \\
\text { moderada } \\
(\%)\end{array}$ & p-valor \\
\hline Tipo de Cuidador & & & & $0,058^{*}$ \\
\hline $\begin{array}{l}\text { Formal } \\
\text { Informal }\end{array}$ & $\begin{array}{r}27(58,7) \\
18(41,3)\end{array}$ & $\begin{array}{l}73,1 \\
26,9\end{array}$ & $\begin{array}{l}40,0 \\
55,0\end{array}$ & \\
\hline Regime de Plantão & & & & $1,000^{*}$ \\
\hline Sim & $24(50,0)$ & 50,0 & 50,0 & \\
\hline Não & $24(50,0)$ & 50,0 & 50,0 & \\
\hline Plantão & & & & $0,020^{* *}$ \\
\hline 8 horas & $02(8,3)$ & 14,3 & - & \\
\hline 12 horas & $18(75,0)$ & 85,7 & 60,0 & \\
\hline 16 horas ou mais & $04(16,7)$ & - & 40,0 & \\
\hline Remuneração & & & & $0,065^{*}$ \\
\hline Sim & $29(60,4)$ & 71,4 & 45,0 & \\
\hline Não & $09(39,6)$ & 28,6 & 55,0 & \\
\hline Vínculo Familiar & & & & $0,024^{*}$ \\
\hline Familiar (Esposa(o)/Filha(o)) & $16(33,3)$ & 17,9 & 55,0 & \\
\hline Nenhum & $32(66,7)$ & 82,1 & 45,0 & \\
\hline Cuidador por Opção & & & & $0,558^{* *}$ \\
\hline Sim & $44(91,7)$ & 92,9 & 90,0 & \\
\hline Não & $04(8,3)$ & 07,1 & 10,0 & \\
\hline Tempo c/ Cuidador & & & & $0,585^{\star *}$ \\
\hline Até 12 meses & $08(16,7)$ & 21,4 & 10,0 & \\
\hline Acima de 12 meses & $40(83,3)$ & 78,6 & 90,0 & \\
\hline Principais Atvs & & & & $0,563^{* *}$ \\
\hline 1 a 3 atividades & $10(21,3)$ & 25,0 & 15,8 & \\
\hline 4 ou mais atividades & $37(78,7)$ & 75,0 & 84,2 & \\
\hline
\end{tabular}


Tabela 2. Domínios da qualidade de vida, sintomas depressivos e nível de atividade física de acordo com a sobrecarga dos cuidadores participantes do estudo. Florianópolis. 2017.

\begin{tabular}{|c|c|c|c|c|}
\hline & $\begin{array}{c}\text { Total } \\
\text { Média }( \pm)\end{array}$ & $\begin{array}{c}\text { Sobrecarga leve ou } \\
\text { ausência } \\
\text { Média }( \pm)\end{array}$ & $\begin{array}{c}\text { Sobrecarga } \\
\text { moderada } \\
\text { Média }( \pm)\end{array}$ & p-valor \\
\hline Domínio Físico & $58,2(10,0)$ & $60,4(8,9)$ & $55,0(10,8)$ & $0,066^{*}$ \\
\hline Domínio Psicológico & $57,3(13,5)$ & $60,5(10,8)$ & $52,9(15,9)$ & $0,054^{*}$ \\
\hline Domínio Social & $68,2(15,4)$ & $70,5(15,9)$ & $65,0(14,4)$ & $0,224^{*}$ \\
\hline Domínio Ambiental & $62,2(15,0)$ & $63,0(15,3)$ & $61,2(15,0)$ & $0,687^{*}$ \\
\hline Sintomas depressivos & $(\%)$ & $(\%)$ & $(\%)$ & \\
\hline $\begin{array}{l}\text { Sintomas depressivos leves } \\
\text { ou ausentes }\end{array}$ & $45(93,8)$ & 100,0 & 85,0 & $0,066^{* *}$ \\
\hline $\begin{array}{l}\text { Sintomas depressivos } \\
\text { moderados }\end{array}$ & $03(6,2)$ & - & 15,0 & \\
\hline \multicolumn{5}{|l|}{ Nível de atividade Física } \\
\hline Insuficientemente ativo & $46(95,8)$ & 58,7 & 41,3 & $0,665^{\star *}$ \\
\hline Ativo & $01(2,1)$ & - & 100,0 & \\
\hline Muito ativo & $01(2,1)$ & 100,0 & - & \\
\hline
\end{tabular}

todos os cuidadores foram relacionados como insuficientemente ativos (96\%).

\section{DISCUSSÃO}

O presente artigo teve como objetivo investigar o nível de atividade física, a qualidade de vida e os sintomas depressivos, de acordo com a sobrecarga de cuidadores de indivíduos com a doença de Parkinson.

Neste estudo, ocorreu o predomínio de cuidadoras do sexo feminino, casadas, com idade média de 45 anos e com o nível de escolaridade médio. Tais características, são frequentemente encontradas em pesquisas em que as esposas ou filhas dos pacientes são as principais cuidadoras ${ }^{14,23}$, tendo também um nível de escolaridade médio ou superior incompleto ${ }^{24}$. Esses resultados do vínculo familiar reforçam o papel social da mulher no aspecto cultural; no qual as mulheres lidam melhor com a tarefa do cuidar do que os homens; o cuidar é uma tarefa difícil e que mexe muito com a estrutura emocional, além do doar-se a uma segunda pessoa $a^{11,16,23,25}$. O baixo nível de escolaridade pode prejudicar na prestação de cuidados oferecidos ao indivíduo, como também, requer atenção redobrada por parte dos cuidadores, pois muitas vezes o cuidador não é um profissional da saúde e apresenta, como limitação, a falta do processo de educação em saúde para tratar o seu familiar ou paciente ${ }^{24}$.

O vínculo familiar e o regime de plantão acima de 12 horas tiveram uma relação próxima da sobrecarga, comparados com os cuidadores que não possuíam vínculo com o indivíduo e não trabalhavam sob o regime de plantão. Isso, talvez, possa ser explicado devido ao aumento da demanda de cuidados, 
ao tempo que o familiar passa em contato com o individuo, as restrições que o cuidador possui na sua vida pessoal, além de ter que conviver com todos os afazeres domésticos e profissionais que lhe são exigidos ${ }^{26,11,14}$.

Os cuidadores que trabalhavam com vínculo empregatício, sem o regime de plantão, mostraram uma menor sobrecarga, podendo estar associado ao cuidador manter o contato apenas no horário de expediente de trabalho e, após esse horário, ele poder continuar a sua rotina normalmente, sem ter que mudar os seus afazeres em função do paciente ${ }^{27}$. Isso é elucidado no estudo de Silva ${ }^{14}$, que avaliou a sobrecarga de cuidadores de pacientes com demência e mostrou que, cuidadores que ficavam na companhia do paciente por mais de 12 horas, apresentavam maiores chances de desenvolver transtornos mentais, maiores queixas de falta de memoria e um maior uso de antidepressivo, reforçando os resultados de sobrecarga por regime de plantão e de vínculo familiar.

É importante salientar, que a sobrecarga pode ser traduzida como um estresse, se manifestando normalmente quando existe um acúmulo de tarefas e conhecimento insuficiente para lidar com essas tarefas ${ }^{27,28}$. O nível alto de sobrecarga do cuidador, geralmente, está associado ao nível de dependência do paciente, ao grau da sua patologia, às condições financeiras, as limitações das suas atividades sociais em prol do paciente, consequentemente, reduzindo a qualidade de vida do cuidador ${ }^{27}$. O aumento da sobrecarga de trabalho dos cuidadores pode ocasionar tensão, ansiedade e sintomas depressivos ${ }^{29}$.

Diante da demanda de cuidados e de múltiplas tarefas desafiadoras, que incluem a aceitação do diagnóstico da doença, administrar os conflitos familiares, reprogramar o futuro, o suporte financeiro, podendo deixar o cuidador formal ou informal vulnerável ao sentimento de depressão ${ }^{14,26}$. Sentimentos de depressão e ansiedade ocorrem quando o cuidador percebe que a sua estrutura familiar está comprometida, devido às modificações ocorridas em relação à doença ${ }^{14}$. No referido estudo não existiram diferenças significativas entre os grupos (sobrecarga leve ou ausente; sobrecarga moderada), porém, destaca-se que os cuidadores com sobrecarga leve ou ausente apresentaram escores mais elevados de qualidade de vida, bem como, maiores percentuais de sintomas depressivos leves ou ausentes. No entanto, a literatura mostra, de uma forma geral, não dividindo em cuidadores formais e não formais, que $60 \%$ a $70 \%$ dos cuidadores têm um alto nível de estresse, de ansiedade, de depressão e um maior sentimento de isolamento ${ }^{10,14}$.

Entretanto, mesmo não sendo significativos neste estudo, os sintomas depressivos foram maiores, assim como, os escores mais baixos de qualidade de vida em cuidadores informais (família). Isso tem muita relação com a dificuldade de aceitação da doença, inversão de papeis, como no caso de pais e filhos e, a responsabilidade da função, que podem estar associadas aos níveis de depressão ${ }^{26}$. Outra variável predisposta a níveis depressivos são as preocupações financeiras, a segurança, o lazer, os cuidados pessoais, visto que o cuidador se preocupa mais com as questões financeiras, para de trabalhar e perde o interesse por atividades pessoais ${ }^{10}$. O fato do cuidador achar que 
ninguém cuidará do indivíduo com DP, tão bem quanto ele e a necessidade de estar sempre presente, faz com que muitos cuidadores não confiem essa atividade a mais nenhuma outra pessoa e, quando sentem a necessidade de "terceirizar" os cuidados, eles são tomados por sentimento de culpa, preocupação e insegurança, fortalecendo ainda mais a sobrecarga física e psicológica do cuidador ${ }^{26}$.

O domínio da $Q V$ mais positivo no estudo foi o domínio social, que está relacionado ao suporte ou apoio que o cuidador recebe de outras pessoas. Os bons resultados no domínio social podem estar associados a um maior número de cuidadores formais no estudo, pois como citado, ter um vínculo empregatício e não manter contato prolongado com o paciente pode reduzir a sobrecarga do cuidador, visto que ele não se envolve mais do que o necessário com o paciente ${ }^{27}$.

A prática da atividade física desempenha um papel importante na promoção da saúde, nos sintomas depressivos e na percepção da $\mathrm{QV}^{14}$. Aatividade física traz inúmeros benefícios tanto nos aspectos físicos e psicológicos dos cuidadores, restaurando o bem-estar físico, social e mental ${ }^{9}$, elevando a autoestima, contribuindo para as relações interpessoais, ajudando no reequilíbrio emocional, na concentração e na memoria, trazendo muito mais disposição ao cuidador ${ }^{14}$. Os cuidadores avaliados foram considerados, praticamente todos, como insuficientemente ativos, o que é um resultado muito preocupante, podendo inferir-se para estes resultados, a falta de tempo por parte do cuidador, considerando que muitos deles, além de cuidarem do seu familiar ou do paciente (quando formal), trabalham fora de casa, possuem filhos para cuidar ou cuidam da casa, ou seja, possuem outras atividades no seu dia a dia e não priorizam a atividade física, devido a uma atividade diária de trabalho exaustivo ${ }^{30}$.

Constituiu-se como limitação deste estudo, o número reduzido de cuidadores, uma vez que não existe registro formal dos mesmos, sem contar a informalidade dos cuidadores familiares, dificultando a ampliação do mesmo. Outra possível limitação pode ser o uso do IPAQ (versão curta), diante das dificuldades de aplicação desse instrumento, pela dificuldade de compreensão das questões, uma vez que se tratam de questões subjetivas ${ }^{31}$, levando a um resultado preocupante de inatividade física por parte dos cuidadores. Diante disto, é necessário que o profissional da saúde volte sua atenção aos cuidadores, verificando as barreiras sociais e físicas envolvidas no ato de cuidar e elaborar estratégias e planejamento de suporte social, bem como, o incentivo a prática de atividade física com o objetivo de diminuir a sobrecarga de trabalho e oferecer ainda mais qualidade de vida a esses cuidadores e a seus familiares.

\section{CONCLUSÃO}

De acordo com as características obtidas, verificou-se que os cuidadores de individuo com a DP eram, predominantemente, do sexo feminino, com idade média de 45 anos e com grau de escolaridade médio. A maioria dos cuidadores estava insuficientemente ativos, porém, com uma boa percepção da qualidade de vida e com baixo sintomas depressivos. A sobrecarga foi significativa, apenas, em cuidadores formais, que realizavam 
plantões acima de 12 horas ou em cuidadores informais, que mantinham contato com o individuo por mais de 12 horas por dia. Mesmo com uma sobrecarga de trabalho elevada, todos os domínios da qualidade de vida obtiveram percentuais positivos, bem como, os sintomas depressivos leves ou ausentes, mostrando uma melhor conscientização do cuidador frente a sua realidade.

\section{REFERENCIAS}

1. Balestrino R, Schapira AH. Parkinson Disease. European journal of neurology, 2019; 27:27-42.

2. Sowalsky KL, Sonke J, Altmann LJP, Almeida L, Hass CJ. Biomechanical Analysis of Dance for Parkinson's Disease: A Paradoxical Case Study of Balance and Gait Effects?. Explore: The J of Science and Healing, 2017; $13: 409-413$

3. Balestrino R, Martinez-Martin P. Neuropsychiatric symptoms, behavioural disorders, and quality of life in Parkinson's disease. J Neurol Sci, 2017;373:173-178.

4. Hirsch L, Jette N, Frolkis A, Steeves T, Pringsheim T. et al. The Incidence of Parkinson's Disease: A Systematic Review and Meta-Analysis. Neuroepidemiology, 2016; 46:292-300.

5. Silva FS, Pabis JVPC, Alencar AG, Silva KB, NavarroPeternella FM, et al. Evolução da doença de Parkinson e comprometimento da qualidade de vida. Rev Neurocienc, 2010; 18:463-468.

6. Dickson, DW. Neuropathology of Parkinson disease. Parkinsonism \& Related Disorders, 2018;46.

7. McNeely ME, Duncan RP, Earhart GM. A comparison of dance interventions in people with Parkinson disease and older adults. Maturitas, 2015; 81:10-6.

8. Bruno AE, Sethares KA. Fatigue in Parkinson disease: an integrative review. J Neurosci Nurs; 2015; 47:146153.

9. Moraes Filho AV, Chaves SN, Martins WR, Tolentino GP, et al. Progressive Resistance Training Improves Bradykinesia, Motor Symptoms and Functional Performance in Patients with Parkinson's Disease, 2020;15:87-95.

10. Tew EH, Naismith SL, Pereira M, Lewis SJG. Quality of Life in Parkinson's Disease Caregivers: The Contribution of Personality Traits. BioMed Research International, 2013; 15:72-78.

11. Filippin NT, Martins JS, Dela Libera LB, Halberstadt BF, Severo AR. Qualidade de vida de sujeitos com doença de Parkinson e seus cuidadores. Fisioter Mov, 2014; 27:57-66.

12. Nunes SFL, Alvarez AM, Valcarenghi RV, Hammerschimit KSA, Baptista R. Adaptação dos Familiares Cuidadores de Idosos com Doença de Parkinson: Processo de Transição. Psicologia: Teoria e Pesquisa, 2019;35.

13. Rezende TC, Coimbra AM, Costallat LT, Coimbra IB, et al. Factors of high impacts on the life of caregivers of disabled elderly. Arch Gerontol Geriatr, 2010; 51:76-80.

14. Silva CF, Passos VMA, Barreto SM. Frequência e repercussão da sobrecarga de cuidadores familiares de idosos com demência. Rev. Bras. Geriatr. Gerontol, 2012; 15:707-731.

15. Billinger SA, et al. Physical Activity and Exercise Recommendations for Stroke Survivors. American Heart Association, 2014; 45:2532-2553.

16. Talhaferro BV, Arakaki IO, Carrasco KG. O Impacto da doença de Alzheimer no familiar cuidador no interior do estado de São Paulo. Psic. Rev, 2015; 24:229-251.

17. Hoehn MM, Yahr MD. Parkinsonism: onset, progression and mortality. Neurology, 1967; 17:427-42.

18. Pardini R, Matsudo S, Matsudo V, Araujo T, Andrade E, Braggion G, et al. Validation of International Physical Questionnaire (IPAQ): pilot study in Brazilian young adults. Med Sci Sports Exerc, 1997; 29:S5-S9.

19. Fleck MP. A. Aplicação da versão em português do instrumento abreviado da qualidade de vida "WHOQOLbref. Revista de Saúde Pública, 2000; 34:178-183.

20. Scazufca M. Versão brasileira da escala Burden Interview para avaliação de sobrecarga em cuidadores de indivíduos com doenças mentais. Rev Bras de Psiquiatria, 2002; 24:12-17.

21. BeckAT, et al. An inventory for measuring depression. Archives of General Psychiatry, 1961; 4:561-71.

22. Cunha JA, et al. Manual da versão em português das Escalas de Beck. Casa do Psicólogo, 2001.

23. Tomomitsu MRSV, Perracini MR, Neri AL. A influência de gênero, idade e renda sobre o bem-estar de idosos cuidadores e não cuidadores. Rev. Bras. Geriatr. Gerontol, 2013; 16:663-680.

24. Santos AA, Pavarini SCI. Perfil dos cuidadores de idosos com alterações cognitivas em diferentes contextos de vulnerabilidade social. Rev Gaúcha Enferm, 2010; 31:115-122.

25. Luzardo AR, Gorini MIPC, Silva APSS. Característica de idosos com doença de Alzheimer e seus cuidadores: uma serie de casos em um serviço de neurogeriatria. Texto Contexto Enferm, Florianópolis, 2006; 15:587-94. 
26. Ferreira DPC, Coriolano MGWS, Lins CCSA. A Perspectiva do cuidador da pessoa com Parkinson: revisão integrativa. Rev. Bras. Geriatr. Gerontol, 2017; 20:103-114

27. Uesugui HM. Cuidadores de Idosos: A realidade de um centro de internação domiciliar, Rondônia, Brasil. [Dissertação]. Universidade de Brasília - Brasília - BR; 2011.

28. Kebbe LM. Rosê LBR, Fiorati RC, Carretta, RYD, et al. Cuidando do familiar com transtorno mental: desafios percebidos pelos cuidadores sobre as tarefas do cuidar. Saúde Debate, 2014; 38:494-505.

29. Muniz EA, Freitas CASL, Oliveira EM, Lacerda MR. Grau de sobrecarga dos cuidadores de idosos atendido em domicílio pela Estratégia Saúde da Família. Rev. Saúde Debate, 2016: 40:172-82.
30. Perlini NMOG, Faro ACM. Cuidar de pessoa incapacitada por acidente vascular cerebral no domicílio: O fazer do cuidador familiar. Rev Esc Enferm, 2005; 39:154-163.

31. Vitorino PVO, Barbosa MA, Sousa ALL, Jardim PCBV Ferreira SSF, et al. Prevalência de estilo de vida sedentário entre adolescentes. Acta Paul Enferm, 2014; 27:166-171.

\section{CORRESPONDÊNCIA}

Jéssica Moratelli, Rua Juan Carlos Manucci, 88

São José - Santa Catarina

CEP 88102220, São José - Santa Catarina, Brasil.

E-mail: jessica.moratelli@hotmail.com 